\title{
Opinionated article
}

\section{Donghun Lee and Jay A. Gupta*}

\section{Perspectives on deterministic control of quantum point defects by scanned probes}

https://doi.org/10.1515/nanoph-2019-0212

Received July 10, 2019; revised September 23, 2019; accepted October 3, 2019

\begin{abstract}
Control over individual point defects in solidstate systems is becoming increasingly important, not only for current semiconductor industries but also for next generation quantum information science and technologies. To realize the potential of these defects for scalable and high-performance quantum applications, precise placement of defects and defect clusters at the nanoscale is required, along with improved control over the nanoscale local environment to minimize decoherence. These requirements are met using scanned probe microscopy in silicon and III-V semiconductors, which suggests the extension to hosts for quantum point defects such as diamond, silicon carbide, and hexagonal boron nitride is feasible. Here we provide a perspective on the principal challenges toward this end, and new opportunities afforded by the integration of scanned probes with optical and magnetic resonance techniques.
\end{abstract}

Keywords: quantum information science; scanned probe microscopy; atom manipulation; nitrogen-vacancy (NV) center; dopant control.

\section{Motivation for controlled creation and placement of quantum defects}

As a small sprinkling of spice can drastically change the color and flavor of cuisine, point defects play crucial roles in tuning the electronic properties of semiconductor devices. For example, even at the parts-per-million level, an impurity species can increase the conductivity of an insulating host by many orders of magnitude. This

*Corresponding author: Jay A. Gupta, Department of Physics, The Ohio State University, Columbus, $\mathrm{OH} 43210$, USA, e-mail: gupta.208@osu.edu. https://orcid.org/0000-0002-3908-7719 Donghun Lee: Department of Physics, Korea University, Seoul 02841, Republic of Korea relies on the introduction of impurity energy states that happen to lie near the conduction or valence band edges of the host. In practice, it is the ability to tune the conductivity by impurities that distinguishes semiconductors from insulators, more so than the magnitude of the energy band gap. The discrete nature of these point defects and their random placement in materials is becoming increasingly important for classical devices such as transistors at the nanoscale [1-3]. Beyond the classical, the quantized electronic and spin states of point defects have drawn considerable interest for quantum science and technology $[2,4,5]$. For example, fluctuation of charge/spin states of point defects in Josephson tunnel junctions is a potential source of decoherence for superconducting quantum bits [6]. While such "qubits" have attracted a recent flurry of interest from industry, enlisting the point defects themselves for quantum technologies offers the long-term potential for better scalability and room temperature operation.

Quantum point defects (QPDs) can serve as qubits, single photon sources, and atomic scale sensors [7-10]. The quantum coherence properties of a variety of solidstate defects were investigated in recent years; principally among them are phosphorus dopants in silicon (Si) [11] and nitrogen-vacancy (NV) centers in diamond [7] with more recent interest in divacancies in silicon carbide (SiC) [8] and defects in two-dimensional hexagonal boron nitride (h-BN) [9]. Among these QPD candidates, diamond $\mathrm{NV}$ centers have attracted great interest due to distinct quantum properties such as optically addressable spin states, long coherence lifetimes, and high fidelity quantum operations, all at room temperature (Figure 1) [7-9]. The associated optical absorption and emission provides a route towards coherent coupling between the defects (i.e. spin qubits) and photons (i.e. flying qubits) which is a critical capability for quantum network applications [12, 13]. The dependence of the NV's orbital and spin states on electric, magnetic, and strain fields makes it useful for various sensing applications such as magnetometry, electrometry, and thermometry [14-16]. Moreover, multi-qubit entanglement necessary for quantum computation can be realized by coupling an NV center with neighboring 
A

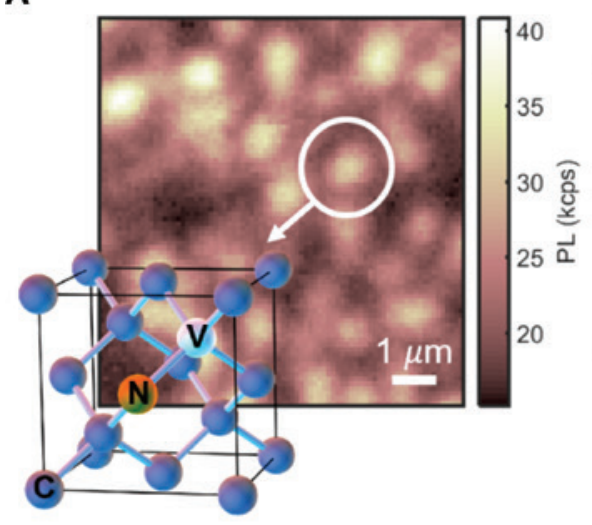

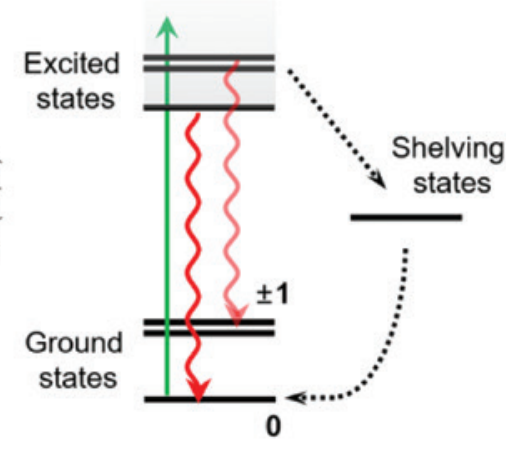

C

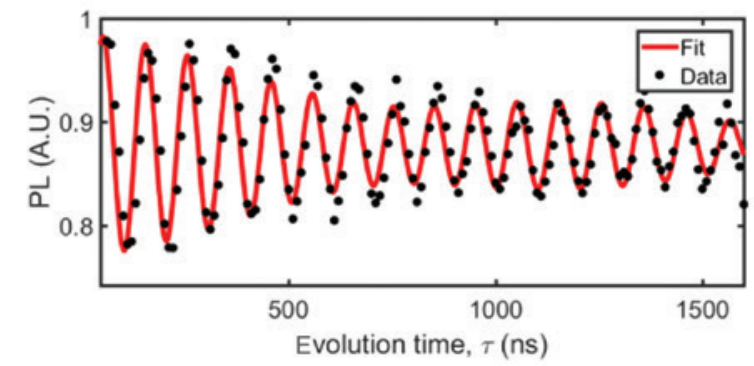

|0〉
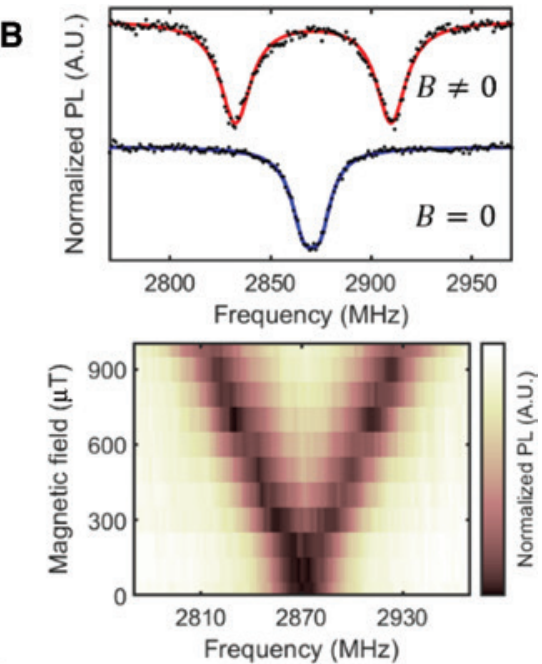

D

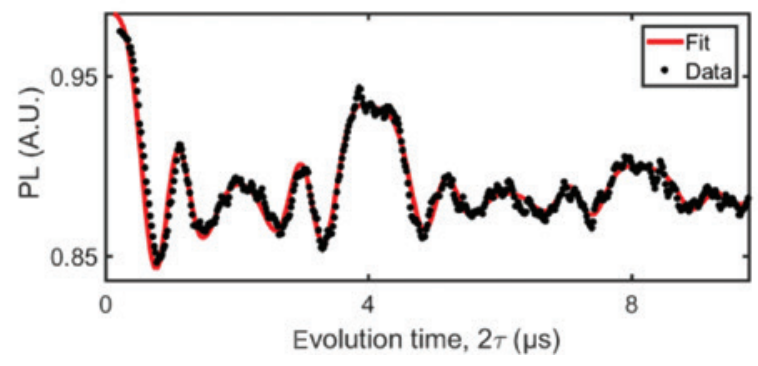

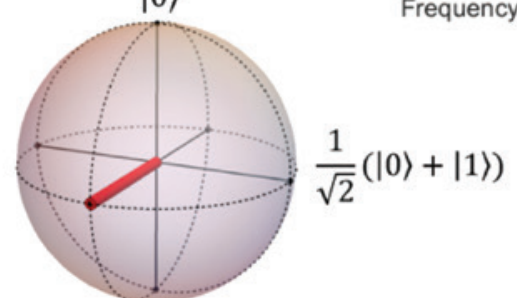

|1)

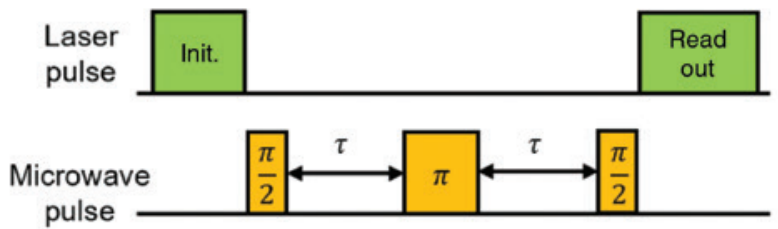

Figure 1: The diamond nitrogen-vacancy (NV) center as a model quantum point defect (QPD).

(A) Confocal image of NV centers and the defect's crystal structure are shown. The energy level diagram displays NV's ground and excited spin states and the relevant optical transitions between them. Due to the dark shelving transitions, the ground spin states can be optically readout and initialized into $m_{\mathrm{s}}=0$ state. (B) Optically detected magnetic resonance as a function of applied magnetic field. Ground $m_{\mathrm{s}}= \pm 1$ spin states split due to the Zeeman effect. (C) Rabi oscillation data show coherent manipulation between two spin states. (D) An example of Hahn spin echo measurements and schematics of laser and microwave pulse sequences. The observed collapses and revivals of the echo signals are due to an applied AC magnetic field at $500 \mathrm{kHz}$.

nuclear spins [17] or other nearby NV centers via magnetic dipole-dipole interactions [18] or indirectly via photons $[12,13]$.

To further build on this foundation, it is desirable to deterministically place QPDs in nanostructures near surfaces or interfaces with nanoscale precision. For instance, the hot spot of diamond photonic structures for strongest coupling to NV centers is a small fraction of the optical wavelength [8], or the diameter of tip apex of diamond NV scanning probe is typically $<100 \mathrm{~nm}$ [15]. As an ultimate goal, magnetic exchange coupling between two QPDs is a direct route to produce quantum entanglement, but this interaction falls off on the scale of the atomic wavefunctions $(<1 \mathrm{~nm})$ [19]. At the same time, the QPD properties must be preserved by keeping them away from edges, surfaces and interfaces, or better yet, controlling fluctuations in this local environment with similar atomic precision.

Various techniques have been developed for placement of QPDs featuring combinations of ion implantation, electron irradiation, and thin film growth. For instance, nitrogen ion implantation introduces vacancies as well, which migrate to the implanted nitrogen sites upon high temperature annealing to generate NV centers at a depth controlled by the implantation energy [20, 21]. Lateral 
control of NV centers is realized by focused ion beams [22] or implantation through fabricated nanoscale apertures $[23,24]$, both methods allowing rastering to form arbitrary nanoscale patterns. Implantation of molecules containing multiple nitrogen atoms (e.g. $\mathrm{N}_{2}, \mathrm{C}_{5} \mathrm{~N}_{4} \mathrm{H}_{n}$ ) improves the chances of generating proximal NV pairs for multi-qubit studies $[25,26]$. Full three-dimensional control can be realized through the combination of delta-doping chemical vapor deposition growth (depth control) and localized vacancy creation with ${ }^{12} \mathrm{C}$ implantation or electron irradiation (lateral control) [27, 28]. Despite these wide-ranging efforts, the current state-of-the-art is still limited to a spatial precision of $\sim 10-100 \mathrm{~nm}[20,21]$ with comparably limited control over the NV's local environment.

\section{Deterministic control of point defects in semiconductors by scanned probes}

Scanned probe techniques provide a promising pathway for deterministic placement and control of QPDs due to the well-established capability for atomic scale manipulation [29]. Scanned probes were used to study defects in semiconductors since the inception of the techniques in the 1980s. Here we will focus on scanning tunneling microscope (STM) studies, which have demonstrated deterministic control of point defects at the atomic scale in both group III-V semiconductors such as GaAs and group IV semiconductors such as $\mathrm{Si}$ and Ge. We will briefly discuss complementary methods, particularly non-contact atomic force microscopy (nc-AFM) in the outlook below.

The (110) surfaces of III-V semiconductors have proven to be a useful family for STM studies of point defects due to the facile cleavage in ultra-high vacuum (UHV) to reveal a pristine, nonpolar surface with relatively small atomic relaxation, and the absence of surface states within the bandgap. These properties reduce Fermi-level pinning and carrier screening, and allow for STM observation of defects down to $\sim 10$ layers below the surface. This spans a regime where the properties of point defects may be distinct from the bulk, and is a model for proximity to interfaces in nanoscale devices. In addition, tunneling spectroscopy can determine defect states in the bandgap, and measure linewidths to estimate lifetimes that may be limited by e.g. coupling to phonons.

Building on prior studies of STM-induced vacancy and adatom motion [30,31], deterministic placement of dopants in a semiconductor with sub-nm precision was first demonstrated by Kitchen et al. for Mn in GaAs [32]. Figure 2 shows the process and examples of deterministic control of point defects at the atomic scale [33]. Starting from a pristine GaAs (110) surface cleaved in UHV, Mn adatoms were deposited in situ at $5 \mathrm{~K}$. The STM tip was positioned over a Mn adatom with negative bias voltage, and the voltage was swept toward positive values while the feedback loop was disabled. Sudden drops in the tunneling current occur when the voltage exceeds $\sim+1 \mathrm{~V}$ and indicate when the substitution occurs. An image of the same area afterwards reveals a new defect, corresponding to substitution of the $\mathrm{Mn}$ adatom for a $\mathrm{Ga}$ atom in the surface layer. The bright dumbbell shape in filled-state imaging reflects the $\mathrm{Mn}$ acceptor's interactions with the neighboring As atoms. This process has been demonstrated for $\mathrm{Co}, \mathrm{Fe}, \mathrm{Er}$, and $V$, all of which are known dopants in GaAs. The ejected Ga adatom from this substitution process has a qualitatively similar appearance to the Mn adatom, and both turn out to be positively charged on the surface. Similar voltage pulses can be used to position charged adatoms or vacancies on the surface, tune defect charge states, or form defect complexes with controlled exchange interactions [32]. Figure 2 shows examples of charge switching of the As vacancy [34], tip-induced ionization of the Mn acceptor [35], tuning by repeated Ga adatom deposition [36], and formation of Mn acceptor complexes with controlled composition, number, spacing, and orientation.

While providing fundamental insights into the origins of magnetic ordering in semiconductors such as $\mathrm{Ga}_{1 x} \mathrm{Mn}_{x} \mathrm{As}$, and forming a basis for proposals in QPD control [37], the potential of these methods for QPD devices has been furthest realized in Si. Commercial Si (100) wafers can be heated in UHV in the presence of atomic hydrogen to produce a clean $(1 \times 1)$ surface where the dangling bonds (DB) associated with surface $\mathrm{Si}$ atoms are occupied by hydrogen atoms. This passivation process is energetically favorable, so that nearly complete passivation of Si surfaces is readily achieved. Tunneling electrons from the STM tip can excite individual hydrogen atoms through a combination of elastic and inelastic processes, thus causing them to desorb from the surface leaving behind an isolated DB [11, 38, 39].

Si DBs represent electronic states which are localized to the atomic scale, and are stable at room temperature. The position and/or occupation of these states represent a binary basis for classical logical operations or nonvolatile storage on atomic length scales. For example, placement of Si DBs into arrays was demonstrated by automated STM [39]. The 0 or 1 of a memory bit is encoded in the presence/absence of a Si DB, so that an array of DBs can 

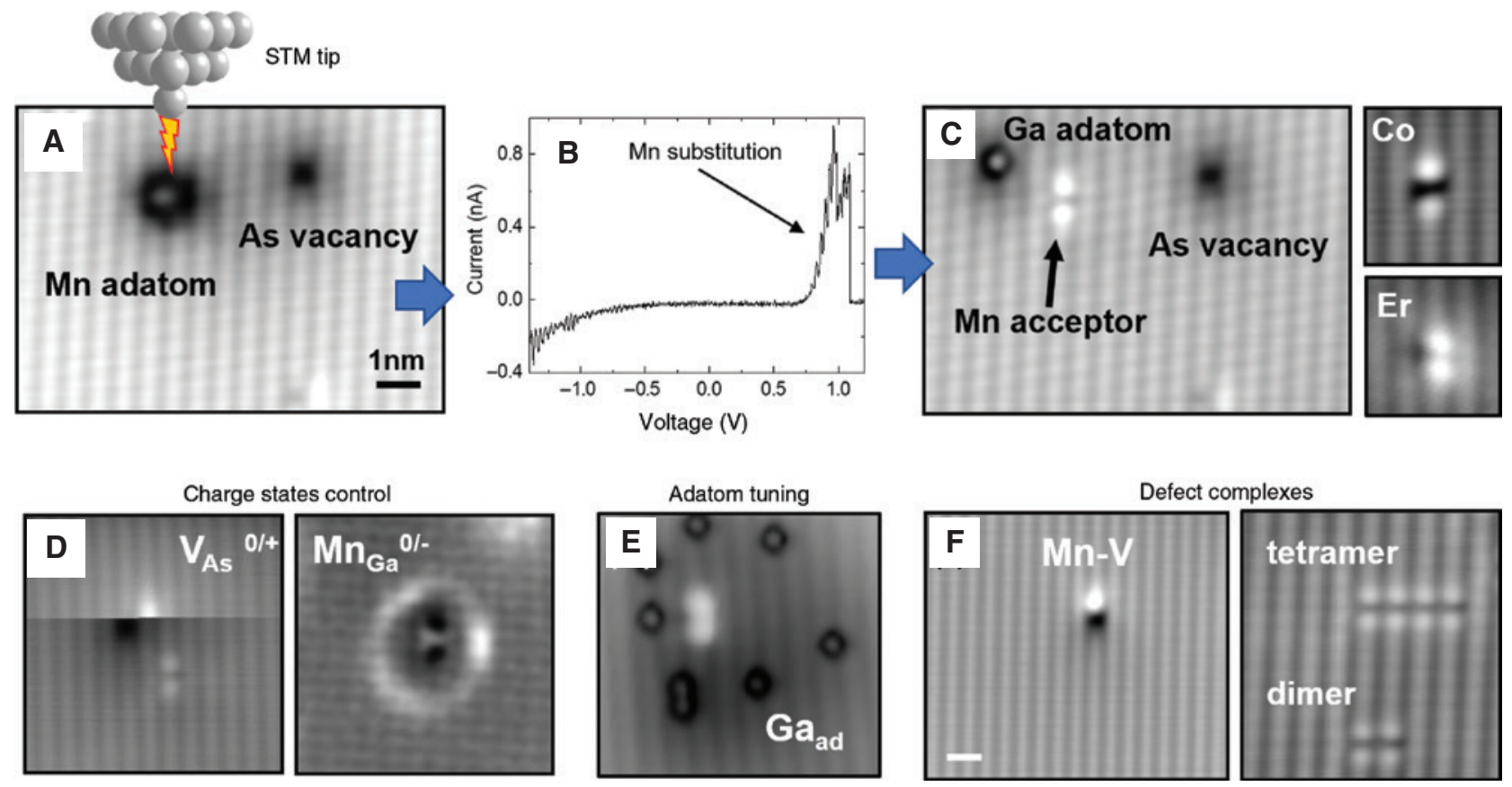

Figure 2: Deterministic control of point defects in a III-V semiconductor by scanning tunneling microscope (STM).

(A) STM image of a Mn adatom and an As vacancy on a GaAs (110) surface. Columns of As atoms are imaged under these conditions, with a spacing of $\sim 560 \mathrm{pm}$. To initiate the substitution process, the STM tip is parked over the Mn adatom, and a positive voltage pulse is applied. (B) Current vs. voltage recorded during a substitution event. (C) STM image after the substitution, showing a Mn acceptor and the ejected Ga adatom. The same process can be applied to other acceptors such as Co and Er. (D) Control over defect charge states. As the tip is scanned over an As vacancy, it switches from a neutral state with bright contrast to a positive charge state with dark contrast. Tip-induced ionization of the neutral $A^{-} h^{+}$acceptor complex to $A^{-}$for the $M n$ acceptor is evident as a ring in maps of the STM $d / / d V$ signal. (E) Tuning of the local electrostatic environment of a Mn acceptor by repeated deposition of charged Ga adatoms from the STM tip. (F) Defect complexes formed using repeated STM atom manipulation.

be patterned to store digital information. The density of such storage is limited essentially only by the atomic $\mathrm{Si}$ lattice itself, and is of order $1 \mathrm{~Pb} / \mathrm{in}^{2}$, which is orders of magnitude greater than the current state-of-the-art. The charge state of coupled DBs can be exploited similarly to form a basis for binary logic relying on the motion of electrons, which is more suitable for fast operation with similarly high density [40].

The STM-induced desorption process also be used to pattern a template into the hydrogen-passivated surface that can control the introduction of dopant species such as phosphorous. Preferential adsorption of molecular species such as $\mathrm{PH}_{3}$ at these sites allows for atomically directed surface chemistry and can be exploited to realize atomically precise placement of impurity atoms [41]. This system has proven suitable for the demonstration of functional quantum electronic devices such as the single-atom transistor, where the active region was patterned with atomic precision [42]. Key to these efforts is the ability to encapsulate the patterned region with additional $\mathrm{Si}$, and address the dopant atoms with electrostatic gates that can also be patterned by coarser-scale STM templating. The coherence properties of phosphorous donors addressed in this way are favorable for quantum information processing, and development of quantum operations is well underway [11, 43].

\section{Outlook for STM studies of QPDs}

The rapidly maturing field of quantum science and technology motivates a sustained and broad effort to translate these STM techniques to deterministic QPD control in diamond, $\mathrm{SiC}, \mathrm{h}-\mathrm{BN}$, and other hosts with long coherence times at room temperature. Principal questions toward this end include: (i) can the coherence properties of bulk-like QPDs be preserved at or near a surface? (ii) is the probe perturbation enough to overcome the larger bond strengths in these materials compared to e.g. Si or III-Vs? (iii) can the relatively stringent conditions for defect control demonstrated so far be relaxed or adapted to these new hosts? and (iv) how can regions patterned on the nanoscale be connected to the outside world? 


\subsection{Surface decoherence}

Typically, the longest coherence times for NV centers (e.g. $T_{2}>1 \mathrm{~ms}$ ) are only observed for defects $>20 \mathrm{~nm}$ below the surface [44-47]. This is attributed to electromagnetic fluctuations due to surface DB or adsorbed species associated with the chemical treatments needed to prepare the samples [45, 46, 48]. Proximity to the surface is desirable to enhance the coupling to nano-photonics and nanomechanical structures, to increase the sensitivity and spatial resolution in QPD-based sensing, and would also make these defects accessible to scanned probes. Great efforts were made to mitigate the surface effects including material treatments for better surface engineering [49] and applications of various quantum control algorithms such as dynamical decoupling and double resonance measurements $[45,46,48]$. These methods have resulted in improved coherence times of $T_{2} \sim 0.1 \mathrm{~ms}$ for NVs only several nanometers below the surface $[45,46,48]$, but this still falls short of the records for bulk NV centers.

\subsection{Probe perturbation}

Despite considerable interest in the physical mechanisms of scanned probe atomic manipulation [50-54], current understanding is largely empirical and limited by the variable terminations of the probes at the nanoscale. For example, in addition to elastic and inelastic electron excitations which are more amenable to theoretical calculations, STM tips have structural variations which influence the nanoscale electric field at the junction, and may also have adsorbed chemical species which influence manipulation. This makes it hard to predict if these methods can be extended to other hosts or how the mechanisms might depend on bond strength without proof-of-principle experiments. Nevertheless, STM is used to dissociate small molecules with comparable bond strengths (e.g. $\mathrm{H}_{2} \mathrm{O}, \mathrm{O}_{2}$ ) to diamond [52], and there is one report of STM-induced desorption of hydrogen-passivated diamond surfaces [55] which suggests that templates for patterned dopant incorporation may be possible.

\subsection{Translation of techniques to QPD hosts}

The wide bandgap of these materials poses a challenge for STM, which requires that the substrate has sufficient conductivity to maintain a steady tunneling current. While there is a long track record of STM studies of SiC, this prior work has mainly focused on the many surface reconstructions and polytypes that are possible in this material [56-58]. To our knowledge there are few reports of point defects, and none using STM manipulation. Diamond is even more challenging to study with STM, but atomic resolution imaging using boron-doped crystals and/or hot-electron injection was demonstrated previously $[59,60]$. There has been, however, relatively little STM work focusing on point defects in diamond. Bulk h-BN has a comparably large band gap as diamond, but is a layered material, which offers new possibilities for access by scanned probes. For example, monolayer and few layer h-BN films thin enough to permit a tunneling current were grown on a variety of metal substrates and studied with STM [61-64]. Recently, a capping layer of graphene was used to probe defects in an underlying $h-B N$ crystal by STM [65]. In this study, charged defects in h-BN could be characterized through their influence on the graphene conductivity, and tip-induced ionization rings were observed as in Figure 2D.

\subsection{Connection to the macroscale}

Additional challenges include QPD control on clean surfaces, stable protection of individual defects after the creation and manipulation, and scalable connection to external macroscopic devices via electrodes and photonic structures. To address these challenges, one may adopt the Si methods discussed above, whereby STM-controlled placement and localization of QPDs is followed by the encapsulation of an epitaxially grown host material and nanoscale registry to alignment markers.

While proof-of-principle demonstrations suggest there are no fundamental barriers, these principal questions remain largely unexplored. In our view, this will require the wider adoption of UHV surface science characterization and growth techniques to QPD systems, and the integration of STM with other imaging, optical, and spin resonance methods (e.g. Figure 3). For example, layerby-layer atom deposition techniques such as molecular beam epitaxy can be incorporated together with STM and other surface science methods (e.g. electron diffraction, Auger spectroscopy), all in a UHV environment. These conditions provide the cleanest possible surfaces to mitigate decoherence and improve QPD accessibility at the surface. To overcome the band gap challenge, STM can be integrated with nc-AFM, which permits comparably high spatial resolution studies of insulating surfaces while maintaining capabilities for atomic manipulation [66]. There has also been remarkable progress in recent years combining STM with in situ optics $[67,68]$ in the visible 


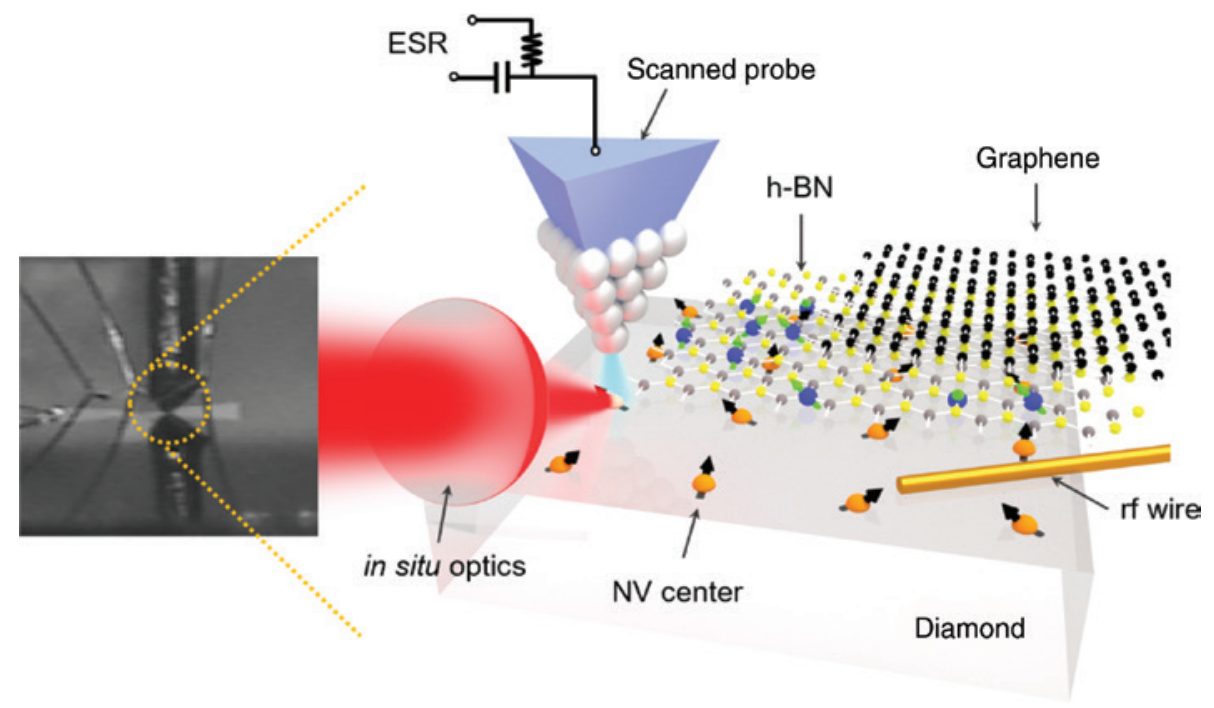

Figure 3: Vision for integrated STM, optics, and magnetic resonance.

The foundation for this effort is an integration of novel device heterostructures with atomic resolution STM imaging and manipulation. Shown in the camera image is an STM tip positioned over electrodes patterned onto a graphene device. Integration with optical excitation and rf excitation allows for deterministic placement and control of individual QPDs. The ability to realize high quality interfaces with 2D materials creates new opportunities for studying coupling between disparate QPDs systems, such as NV centers in diamond and color centers in h-BN.

to $\mathrm{THz}$ regime with ultrafast time resolution [69-71] and with electron/nuclear spin resonance excitation via spin polarized tips [72]. These methods will allow for direct measurement of for example coherence times in concert with atomic-scale characterization of the local environment and possible decoherence pathways. There are now a handful of commercial vendors who sell advanced scanned probe microscope (SPM) systems with these integrated capabilities, which will help broaden the research base beyond specialists with custom-built equipment.

Extension of these methods to NV centers and other QPD systems has the potential benefit that coherent control methods demonstrated with for example optically detected magnetic resonance detection can be performed on QPDs deterministically placed with atomic precision by SPM. Precise defect formation is possible by controlling defect's position, orientation, charge states, and relative separation between pairs or arrays. The same methods can be applied to tune the defect's local environment such as strain, magnetic, and electric field. In this way, one can study the influence of local environment on the defect's quantum coherence properties, thus providing a new opportunity for quantum bath engineering.

Given the disparate and often contradictory requirements for QPDs, we believe there is great potential for deterministically coupling a diverse set of QPDs such as the NV center in diamond and analogous defects in SiC and h-BN or other hosts with optimal quantum properties for storage, operation, and communication. For example, surface adatoms or DB can be used as "quantum reporters" of coherences stored in long-lifetime subsurface QPDs. As an initial step in this direction, paramagnetic spins on diamond surfaces were used to probe and deliver local magnetic information to shallow NV centers [73]. These reporter spins, however, were largely unknown and randomly positioned. Employing the deterministic SPM control methods and using well-identified adatoms/DB, therefore, is expected to provide more effective reporter networks and to enhance the sensitivity and spatial resolution in sensing applications. This approach is particularly powerful for the studies of van der Waals heterostructures containing disparate QPDs. For instance, diamond can be stacked with various 2D materials such as graphene, h-BN, and transition metal dichalcogenide materials [74] which are more readily accessible to deterministic STM techniques. An almost infinite variety of QPD combinations are possible to realize the most effective platforms for the research of multi-qubit quantum information and computation.

In summary, QPDs in solids are promising candidates for future quantum applications but precise placement of defects in desired locations remains a key challenge for scalability. We have given a perspective on the prospects for deterministic control of QPDs using scanned probe methods. We believe the main challenges toward this end can be met using an approach integrating STM with 
other scanned probe, optical, and magnetic resonance techniques. This effort will also build bridges between researchers focusing on advanced scanned probe manipulation of idealized model systems, materials defect researchers who usually rely on an arsenal of ensemble characterization techniques, and the rapidly expanding field of quantum information science.

Acknowledgments: D.L. acknowledges support from the KIST Open Research Program (Project No. 2E2958019-145), the National Research Foundation of Korea (NRF) funded by the Ministry of Science and ICT (NRF-2018R1A4A1024157). J.A.G. acknowledges support from the U.S. Department of Energy under grant \# DE-SC0016379.

\section{References}

[1] Shinada T, Okamoto S, Kobayashi T, Ohdomari I. Enhancing semiconductor device performance using ordered dopant arrays. Nature 2005;437:1128-31.

[2] Koenraad PM, Flatté ME. Single dopants in semiconductors. Nat Mater 2011;10:91-100.

[3] Georgiev VP, Towie EA, Asenov A. Impact of precisely positioned dopants on the performance of an ultimate nanowire transistor: a full three-dimensional NEGF simulation study. IEEE Trans Elect Devices 2013;60:965-71.

[4] Kane BE. A silicon-based nuclear spin quantum computer. Nature 1998;393:133-7.

[5] Weber JR, Koehl WF, Varley JB, et al. Quantum computing with defects. Proc Natl Acad Sci 2010;107:8513-8.

[6] Martinis JM, Cooper KB, McDermott R, et al. Decoherence in Josephson qubits from dielectric loss. Phys Rev Lett 2005;95:210503.

[7] Awschalom DD, Hanson R, Wrachtrup J, Zhou BB. Quantum technologies with optically interfaced solid-state spins. Nat Photon 2018;12:516-27.

[8] Atatüre M, Englund D, Vamivakas N, Lee S-Y, Wrachtrup J. Material platforms for spin-based photonic quantum technologies. Nat Rev Mater 2018;3:38.

[9] Aharonovich I, Englund D, Toth M. Solid-state single-photon emitters. Nat Photon 2016;10:631.

[10] Pla JJ, Tan KY, Dehollain JP, et al. Single-atom electron spin qubit in silicon. Nature 2012;489:541-5.

[11] Zwanenburg FA, Dzurak AS, Morello A, et al. Silicon quantum electronics. Rev Mod Phys 2013;85:961-1019.

[12] Hensen B, Bernien H, Dréau AE, et al. Loophole-free Bell inequality violation using electron spins separated by 1.3 kilometres. Nature 2015;26:682-6.

[13] Kalb N, Reiserer AA, Humphreys PC, et al. Entanglement distillation between solid-state quantum network nodes. Science 2017;356:928-32.

[14] Le Sage D, Arai K, Glenn DR, et al. Optical magnetic imaging of living cells. Nature 2013;496:486.
[15] Gross I. Akhtar W, Garcia V, et al. Real-space imaging of non-collinear antiferromagnetic order with a single-spin magnetometer. Nature 2017;549:252.

[16] Kucsko G, Maurer PC, Yao NY, et al. Nanometre-scale thermometry in a living cell. Nature 2013;500:54-8.

[17] Bradley CE, Randall J, Abobeih MH, et al. A 10-qubit solid-state spin register with quantum memory up to one minute. Phys Rev X 2019;9:031045.

[18] Dolde F, Fedder H, Doherty MW, et al. Electric-field sensing using single diamond spins. Nat Phys 2011;7:459-63.

[19] Kortan VR, Sahin C, Flatte ME. Nanometer-scale exchange interactions between spin centers in diamond. Phys Rev B 2016;93:220402.

[20] Johnson S, Dolan PR, Smith JM. Diamond photonics for distributed quantum networks. Prog Quant Electron 2017;55:29-65.

[21] Haque A, Sumaiya S. An overview on the formation and processing of nitrogen-vacancy photonic centers in diamond by ion implantation. J Manuf Mater Process 2017;1:6.

[22] Lesik M, Spinicelli P, Pezzagna S, et al. Maskless and targeted creation of arrays of colour centres in diamond using focused ion beam technology. Phys Status Solidi A 2013;210:2055-9.

[23] Toyli DM, Weis CD, Fuchs GD, Schenkel T, Awschalom DD. Chip-scale nanofabrication of single spins and spin arrays in diamond. Nano Lett 2010;10:3168-72.

[24] Spinicelli P, Dréau A, Rondin L, et al. Engineered arrays of nitrogen-vacancy color centers in diamond based on implantation of $\mathrm{CN}$-molecules through nanoapertures. New J Phys 2011;13:025014.

[25] Yamamoto T, Müller C, McGuinness LP, et al. Strongly coupled diamond spin qubits by molecular nitrogen implantation. Phys Rev B 2013;88:20120.

[26] Haruyama M, Onoda S, Higuchi T, et al. Triple nitrogen-vacancy centre fabrication by $\mathrm{C} 5 \mathrm{~N} 4 \mathrm{H} \mathrm{n}$ ion implantation. Nat Commun 2019;10:2664.

[27] Ohno K, Joseph Heremans F, de las Casas CF, et al. Threedimensional localization of spins in diamond using $12 \mathrm{C}$ implantation. Appl Phys Lett 2014;105:052406.

[28] McLellan CA, Myers BA, Kraemer S, Ohno K, Awschalom DD, Bleszynski JAC. Patterned formation of highly coherent nitrogen-vacancy centers using a focused electron irradiation technique. Nano Lett 2016;16:2450-4.

[29] Eigler D, Schweizer E. Positioning single atoms with a scanning tunneling microscope. Nature 1990;344:524-6.

[30] Lengel G, Harper J, Weimer M. Charge injection and STMinduced vacancy migration on GaAs(110). Phys Rev Lett 1996;76:4725-8.

[31] Whitman LJ, Stroscio JA, Dragoset RA, Celotta RJ. Manipulation of adsorbed atoms and creation of new structures on roomtemperature surfaces with a scanning tunneling microscope. Sci New Ser 1991;25:1206-10.

[32] Kitchen D, Richardella A, Tang JM, Flatté ME, Yazdani A. Atomby-atom substitution of $\mathrm{Mn}$ in GaAs and visualization of their hole-mediated interactions. Nature 2006;442:436-9.

[33] Lee D, Gohlke D, Benjamin A, Gupta JA. Influence of the local environment on Mn acceptors in GaAs. J Phys Condens Matter 2015;27:154202.

[34] Lee DH, Gupta JA. Tunable field control over the binding energy of single dopants by a charged vacancy in GaAs. Science 2010;330:1807-10. 
[35] Lee DH, Gupta JA. Tunable control over the ionization state of single $\mathrm{Mn}$ acceptors in GaAs with defect-induced band bending. Nano Lett 2011;11:2004-7.

[36] Gohlke D, Mishra R, Restrepo OD, Lee D, Windl W, Gupta J. Atomic-scale engineering of the electrostatic landscape of semiconductor surfaces. Nano Lett 2013;13:2418-22.

[37] Tang JM, Levy J, Flatté ME. All-electrical control of single ion spins in a semiconductor. Phys Rev Lett 2006;97:106803.

[38] Shen TC, Wang C, Abeln GC, et al. Atomic-scale desorption through electronic and vibrational excitation mechanisms. Science 1995;268:1590-2.

[39] Achal R, Rashidi M, Croshaw J, et al. Lithography for robust and editable atomic-scale silicon devices and memories. Nat Commun 2018;9:2778.

[40] Labidi H, Koleini M, Huff T, et al. Indications of chemical bond contrast in AFM images of a hydrogen-terminated silicon surface. Nat Commun 2017;8:14222.

[41] Rueß FJ, Pok W, Reusch TCG, et al. Realization of atomically controlled dopant devices in silicon. Small 2007;3:563-7.

[42] Fuechsle M, Miwa JA, Mahapatra S, et al. A single-atom transistor. Nat Nanotechnol 2012;7:242-6.

[43] Salfi J, Mol JA, Rahman R, et al. Quantum simulation of the Hubbard model with dopant atoms in silicon. Nat Commun 2016;7:11342.

[44] Balasubramanian G, Neumann P, Twitchen D, et al. Ultralong spin coherence time in isotopically engineered diamond. Nat Mater 2009;8:383

[45] Romach Y, Müller C, Unden T, et al. Spectroscopy of surfaceinduced noise using shallow spins in diamond. Phys Rev Lett 2015;114:01760.

[46] Kim M, Mamin HJ, Sherwood MH, Ohno K, Awschalom DD, Rugar D. Decoherence of near-surface nitrogen-vacancy centers due to electric field noise. Phys Rev Lett 2015;115:087602.

[47] Bluvstein D, Zhang Z, Jayich ACB. Identifying and mitigating charge instabilities in shallow diamond nitrogen-vacancy centers. Phys Rev Lett 2019;122:07610.

[48] Bluvstein D, Zhang Z, McLellan CA, Williams NR, Jayich ACB. Extending the quantum coherence of a near-surface qubit by coherently driving the paramagnetic surface environment. Phys Rev Lett 2019;123:146804.

[49] Tisler J, Balasubramanian G, Naydenov B, et al. Fluorescence and spin properties of defects in single digit nanodiamonds. ACS Nano 2009;3:1959-65.

[50] Avouris P. Manipulation of matter at the atomic and molecularlevels. Acc Chem Res 1995;28:95-102.

[51] Tsong T. Effects of an electric-field in atomic manipulations. Phys Rev B 2002;44:13703-10.

[52] Ho W. Single-molecule chemistry. J Chem Phys 2002;117:11033-61.

[53] Lyo I, Avouris P. Field-induced nanometer- to atomic-scale manipulation of silicon surfaces with the STM. Sci Wash 1991;253:173.

[54] Hofer WA, Foster AS, Shluger AL. Theories of scanning probe microscopes at the atomic scale. Rev Mod Phys 2003;75:1287-331.
[55] Bobrov K, Mayne AJ, Hoffman A, Dujardin G. Atomic-scale desorption of hydrogen from hydrogenated diamond surfaces using the STM. Surf Sci 2003;528:138-43.

[56] Owman F, Martensson P. STM study of the Sic(0001)root-3xRoot-3 surface. Surf Sci 1995;330:L639-45.

[57] Li L, Tsong IST. Atomic structures of $6 \mathrm{H}-\mathrm{SiC}(0001)$ and (0001) surfaces. Surf Sci 1996;351:141-8.

[58] Soukiassian P, Semond F, Douillard L, et al. Direct observation of a beta-SiC(100)-c(4×2) surface reconstruction. Phys Rev Lett 1997;78:907-10.

[59] Bobrov K, Mayne AJ, Dujardin G. Atomic-scale imaging of insulating diamond through resonant electron injection. Nature 2001;413:616-9.

[60] Nimmrich M, Kittelmann M, Rahe P, et al. Atomic-resolution imaging of clean and hydrogen-terminated $C(100)-(2 \times 1)$ diamond surfaces using noncontact AFM. Phys Rev B 2010;81:201403(R).

[61] Auwarter W, Kreutz TJ, Greber T, Osterwalder J. XPD and STM investigation of hexagonal boron nitride on $\mathrm{Ni}(111)$. Surf Sci 1999;429:229-36.

[62] Lee JS, Choi SH, Yun SJ, et al. Wafer-scale single-crystal hexagonal boron nitride film via self-collimated grain formation. Science 2019;362:817-21

[63] Li Q, Zou X, Liu M, et al. Grain boundary structures and electronic properties of hexagonal boron nitride on $\mathrm{Cu}(111)$. Nano Lett 2015;15:804-10.

[64] Schulz F, Drost R, Hamalainen SK, Demonchaux T, Seitsonen AP, Liljeroth P. Epitaxial hexagonal boron nitride on $\operatorname{Ir}(111)$ : a work function template. Phys Rev B 2014;89:235429.

[65] Wong D, Velasco J, Ju L, et al. Characterization and manipulation of individual defects in insulating hexagonal boron nitride using scanning tunnelling microscopy. Nat Nanotechnol 2015;10:949-53.

[66] Giessibl FJ. The qPlus sensor, a powerful core for the atomic force microscope. Rev Sci Instrum 2019;90:011101.

[67] Grafstrom S. Photoassisted scanning tunneling microscopy. J Appl Phys 2002;91:1717-53.

[68] Kuhnke K, Grosse C, Merino P, Kern K. Atomic-scale imaging and spectroscopy of electroluminescence at molecular interfaces. Chem Rev 2017;117:5174-222.

[69] Terada Y, Yoshida S, Takeuchi O, Shigekawa H. Real-space imaging of transient carrier dynamics by nanoscale pumpprobe microscopy. Nat Photon 2010;4:869-74.

[70] Cocker TL, Jelic V, Gupta M, et al. An ultrafast terahertz scanning tunnelling microscope. Nat Photon 2013;7:620-5.

[71] Li S, Chen S, Li J, Wu R, Ho W. Joint space-time coherent vibration driven conformational transitions in a single molecule. Phys Rev Lett 2017;119:176002.

[72] Willke P, Paul W, Natterer FD, et al. Probing quantum coherence in single-atom electron spin resonance. Sci Adv 2018;4:eaaq1543.

[73] Sushkov AO, Lovchinsky I, Chisholm N, Walsworth RL, Park H, Lukin MD. Magnetic resonance detection of individual proton spins using quantum reporters. Phys Rev Lett 2014;113:197601.

[74] Geim AK, Grigorieva IV. Van der Waals heterostructures. Nature 2013;499:419-25. 